\title{
EFECTOS ECOTOXICOLOGICOS DEL PETROLEO CRUDO, DIESEL 2 Y KEROSENE SOBRE EL CRECIMIENTO POBLACIONAL DE LA MICROALGA Chaetoceros gracilis Schutt
}

\section{ECOTOXICOLOGICAL EFFECTS OF CRUDE OIL, DIESEL 2 AND KEROSENE ON THE POPULATION GROWTH OF THE MICROALGAE Chaetoceros gracilis Schutt}

\author{
Giovanna Vera ${ }^{1}$, Jorge Tam $^{2}$ y Edwin Pinto ${ }^{3}$
}

\begin{abstract}
Resumen
Se realizaron pruebas ecotoxicológicas para determinar los efectos del petróleo crudo, Diesel 2 y kerosene sobre el crecimiento poblacional de la diatomea Chaetoceros gracilis. Se determinó la concentración efectiva media $\left(\mathrm{CE}_{50 \%}\right)$ a un tiempo de exposición de 96 h, siendo más tóxica la solución con Diesel $2\left(90 \mathrm{mg} \cdot \mathrm{L}^{-1}\right)$, seguida de la solución con kerosene $\left(98 \mathrm{mg} \cdot \mathrm{L}^{-1}\right)$ y la solución con petróleo crudo (867.5 mg. $\mathrm{L}^{-1}$ ). Además, se evaluó los efectos ocasionados en la tasa intrínseca del crecimiento poblacional y en la tasa de división de la especie expuesta a los tres compuestos orgánicos.
\end{abstract}

Palabras clave: Chaetoceros gracilis, crecimiento poblacional, hidrocarburos de petróleo, pruebas ecotoxicológicas

\begin{abstract}
Ecotoxicological tests were carried out to determine the effects of crude oil, Diesel 2 and kerosene on the population growth of the diatom Chaetoceros gracilis. The mean effective concentration $\left(\mathrm{CE}_{50 \%}\right)$ at an exposure time of $96 \mathrm{~h}$ was determined. The solution with Diesel 2 was more toxic $\left(90 \mathrm{mg} \cdot \mathrm{L}^{-1}\right)$, followed by the solution with kerosene $\left(98 \mathrm{mg} \cdot \mathrm{L}^{-1}\right.$ ) and the solution with crude oil $\left(867.5 \mathrm{mg} \cdot \mathrm{L}^{-1}\right)$. In addition, the effects of the three organic compounds on the intrinsic population growth rate and division rate of $C h$. gracilis were assessed.

Key words: Chaetoceros gracilis, ecotoxicological bioassays, oil hydrocarbons, population growth
\end{abstract}

\section{Introducción.}

Una de las fuentes más importantes de contaminación marina en el Perú son los hidrocarburos de petróleo y sus derivados, especialmente durante el cabotaje. En 1990, se derramaron 14000 barriles de kerosene y 438 barriles de petróleo en 1995 (Sánchez \& Orozco, 1997). Otros registros que datan de 1995 al 2001 sobre derrames de petróleo en nuestro mar fueron publicados por Vizcarra (2002).

En el Perú, existen 39 lotes con contratos vigentes para operaciones petroleras, de las cuales 8 se encuentran distribuidas en la zona norte del mar peruano, abarcando los departamentos de Tumbes, Piura, Lambayeque, La Libertad y Ancash con las siguientes cuencas: Cuenca Progreso, Cuenca Lancones, Cuenca Talara , Cuenca Sechura, Cuenca Salaverry. Los otros lotes se encuentran distribuídos al interior del país. También se encuentran 6 plantas de refinamiento: Talara (Piura), La Pampilla (Lima), Conchán (Lima), El Milagro (Amazonas), Pucallpa (Ucayali) e Iquitos (Loreto), así como también 4 plantas de fraccionamiento: Pluspetrol (Ica), Pariña y EEPSA (Piura) y Aguaytía (Ucayali). En la costa peruana existen 18 plantas de abastecimiento: Talara, Piura, Chiclayo (Eten), Trujillo (Salaverry), Chimbote, Supe, Lima (9 plantas), Pisco, Mollendo e Ilo (MEM, 2006).

El Instituto del Mar del Perú (IMARPE) ha realizado diversas evaluaciones de hidrocarburos de petróleo en las zonas de Paita, Talara, Bayóvar, Chimbote, Supe-Paramonga, Callao y Paracas encontrándose en muchos casos altas concentraciones de hidrocarburos en el mar (Guzmán, 1995; Jacinto y Cabello 1996, Jacinto et al. 1996, 1997, 1998; Guzmán et al., 1997 y Cabello et al., 1999).

El primer impacto al producirse un derrame de hidrocarburo ocurre con el fitoplancton, debido a que los hidrocarburos forman una capa impermeable que obstaculiza el paso de la luz solar, fuente necesaria para realizar el proceso fotosintético. Las microalgas unicelulares cumplen un rol esencial en el normal funcionamiento de los ecosistemas marinos, ya que como productores primarios, son el primer eslabón de la cadena alimentaria, oxigenando el agua y participando en los ciclos biogeoquímicos de sustancias orgánicas e inorgánicas. Conocer los efectos ecotoxicológicos de los contaminantes de tipo 
orgánico a través de datos obtenidos en las pruebas de toxicidad usando especies sensibles, ayudará a predecir los posibles efectos sobre las poblaciones $\mathrm{y}$ comunidades algales.

En el Perú, existen pocos estudios acerca del efecto de los hidrocarburos sobre los organismos marinos nativos

(Ibañez \& Huanes, Tabla 1. Composición del Medio de cultivo f/2 (Guillard, 1975 en González et al., 1999 y Alayo \& 1995).

\begin{tabular}{|c|c|c|c|c|c|c|}
\hline $\begin{array}{l}\text { Iannacone, 2002). En } \\
\text { el presente trabajo, se }\end{array}$ & Reactivos & $\begin{array}{l}\text { Volumen } \\
\left(\mathrm{mL}^{-1} \mathrm{~L}^{-1}\right)\end{array}$ & Nutrientes & $\begin{array}{l}\text { Stock } \\
\left(\mathrm{g} . \mathrm{L}^{-1}\right) \\
\end{array}$ & $\begin{array}{l}\text { Medio } \\
\left(\mathrm{g} . \mathrm{L}^{-1}\right) \\
\end{array}$ & Medio $\left(m^{\prime} . L^{-1}\right)$ \\
\hline $\begin{array}{lr}\text { igio } & \text { como } \\
\text { rganismo prueba la }\end{array}$ & Solución 1 & 1 & $\mathrm{NaNO}_{3}$ & 75 & 0.075 & $8.83 \times 10^{-4}$ \\
\hline $\begin{array}{l}\text { atomea } \\
\text { hateoceros gracilis }\end{array}$ & Solución 2 & 1 & $\mathrm{NaH}_{2} \mathrm{PO}_{4}$ & 5 & 0.05 & $3.63 \times 10^{-5}$ \\
\hline $\begin{array}{l}\text { hutt por ser de fácil } \\
\text { anejo }\end{array}$ & Solución 3 & 1 & $\mathrm{Na}_{2} \mathrm{SiO}_{4} \cdot 9 \mathrm{H}_{2} \mathrm{O}$ & 10 & 0.01 & $3.25 \times 10^{-5}$ \\
\hline $\begin{array}{l}\text { boratorio, tamaño } \\
\text { queño, y ciclo de }\end{array}$ & Solución 4 & 1 & $\mathrm{FeCl}_{3} \cdot 6 \mathrm{H}_{2} \mathrm{O}$ & 3.15 & 0.00315 & $1.16 \times 10^{-5}$ \\
\hline $\begin{array}{lr}\text { da } & \text { corto, } \\
\text { Iracterísticas } & \text { que }\end{array}$ & & 955 & $\mathrm{Na}_{2}$ EDTA. $2 \mathrm{H}_{2} \mathrm{O}$ & 4.36 & 0.00436 & $1.17 \times 10^{-1}$ \\
\hline $\begin{array}{l}\text { n ventajosas para la } \\
\text { nducción de }\end{array}$ & & 1 & Mn. $\mathrm{Cl}_{2} \cdot 4 \mathrm{H}_{2} \mathrm{O}$ & $18 \mathrm{~g} .100 \mathrm{~mL}^{-1}$ & 0.00018 & $9.10 \times 10^{-7}$ \\
\hline $\begin{array}{l}\text { uebas } \\
\text { otoxicológicas. }\end{array}$ & & 1 & ZnSo4. 7H2O & $2.2 \mathrm{~g} .100 \mathrm{~mL}^{-1}$ & 0.000022 & $7.65 \times 10^{-8}$ \\
\hline $\begin{array}{ccc}\text { ta } & \text { especie ya ha } \\
\text { do usada como }\end{array}$ & & 1 & CoCl2. $6 \mathrm{H} 2 \mathrm{O}$ & 1.05 g. $100 \mathrm{~mL}^{-1}$ & 0.0000105 & $4.42 \times 10^{-8}$ \\
\hline $\begin{array}{lr}\text { ganismo } & \text { prueba en } \\
\text { os } & \text { estudios }\end{array}$ & & 1 & $\mathrm{CuSO}_{4} \cdot 5 \mathrm{H}_{2} \mathrm{O}$ & 0.98 g. $100 \mathrm{~mL}^{-1}$ & 0.0000098 & $3.93 \times 10^{-8}$ \\
\hline $\begin{array}{l}\text { otoxicológicos } \\
\text { era et al } 200\end{array}$ & & 1 & $\mathrm{Na}_{2} \mathrm{MoO}_{4} \cdot 2 \mathrm{H}_{2} \mathrm{O}$ & 0.63 g. $100 \mathrm{~mL}^{-1}$ & 0.0000063 & $2.62 \times 10^{-8}$ \\
\hline
\end{tabular}

(Vera et al., 2001, Alayo et al., 2004).

Chaetoceros gracilis es una especie que forma parte de la flora fitoplanctónica del mar peruano (Ochoa et al., 1999), y los efectos ecotoxicológicos de las concentraciones de hidrocarburos presentes en las aguas marinas, son transmitidos a niveles tróficos superiores.

Por tales motivos, se planteó como objetivo de la presente investigación evaluar los efectos ecotoxicológicos de los hidrocarburos de petróleo (petróleo crudo, el Diesel 2 y el kerosene) sobre el crecimiento poblacional de la diatomea Chaetoceros gracilis y determinar la concentración efectiva media (CE50\%).

\section{Materiales y métodos.}

Material biológico

La diatomea $C h$. gracilis se obtuvo del Cepario del Laboratorio de la Línea de Investigaciones en Ecotoxicología Acuática del Instituto del Mar del Perú (IMARPE). Esta alga es usada habitualmente como alimento de organismos reproductores de bivalvos y crustáceos en laboratorio, cuyas larvas se utilizan en las diferentes pruebas ecotoxicológicas.

\section{Cultivo de la microalga}

Las cepas de $C h$. gracilis se mantuvieron en fase líquida en un medio Guillard "f/2" modificado (Guillard, 1975 en González et al., 1995). La composición del medio de cultivo utilizado se presenta en la Tabla 1. 
resumen de las condiciones de la prueba ecotoxicológica se presenta en la Tabla 2.

Tabla 2. Condiciones de las pruebas ecotoxicológicas usando Chaetoceros gracilis expuesta a soluciones de petróleo crudo, Diesel 2 y kerosene.

\begin{tabular}{lc} 
Organismo Prueba & Chaetoceros gracilis \\
\hline $\begin{array}{l}\text { Tipo de Prueba } \\
\text { Agua de dilución }\end{array}$ & $\begin{array}{c}\text { Estática, } 96 \mathrm{~h} \\
\text { Agua de mar filtrada estéril y } \\
\text { saturada de oxígeno }\left(6-7 \mathrm{mg} \cdot \mathrm{L}^{-1}\right)\end{array}$ \\
Temperatura & $20^{\circ} \mathrm{C} \pm 1^{\circ} \mathrm{C}$. \\
Fotoperiodo & $\mathrm{L}: \mathrm{O}=24: 0$ \\
Tamaño de matraces & $250 \mathrm{~mL}$ \\
Volumen de las & $150 \mathrm{~mL}$ \\
soluciones & 30000 cél·mL ${ }^{-1}$. \\
Número de células & \\
por matraz & 2 \\
Número de réplicas & \\
por concentración. & Tasa de agitación \\
Agua de dilución & Medio de cultivo f/2 Guillard \\
Concentraciones de & Mínimo 6 y un control \\
prueba & Inhibición del crecimiento \\
Punto final de la & poblacional $(\mathrm{CE} \%)$ \\
prueba &
\end{tabular}

\section{Análisis de datos}

El diseño experimental en las tres pruebas, comprendió 7 tratamientos (6 concentraciones +1 control) y 2 repeticiones por cada tratamiento. La relación dosis-respuesta se obtuvo a partir de los datos de concentración como variable independiente y porcentaje de inhibición como variable dependiente. El porcentaje de inhibición del crecimiento poblacional fue calculado a 96 h de exposición, usando la siguiente fórmula (Joubert, 1980):

$$
I=100\left(1-\frac{N e}{N c}\right)
$$

Donde:

I = Inhibición del crecimiento poblacional (\%)

$\mathrm{Ne}=$ Densidad celular expuesta al tóxico $\left(\right.$ cél $\left.\cdot \mathrm{mL}^{-1}\right)$

$\mathrm{Nc}=$ Densidad celular del control $\left(\right.$ cél $\left.\cdot \mathrm{mL}^{-1}\right)$

Con los datos de inhibición se determinó la concentración efectiva media $\left(\mathrm{CE}_{50 \%}\right)$ utilizando el programa computacional PROBIT (Weber, 1993), que calcula la concentración de hidrocarburo que determina una inhibición del crecimiento poblacional del $50 \%$.

Adicionalmente, se estimó la tasa intrínseca de crecimiento poblacional (r) y la tasa de división (D). La tasa intrínseca de crecimiento poblacional se estimó del modelo logístico contínuo, usando la siguiente fórmula (Franco, 1985):

$$
N_{t}=\frac{K}{(1+\exp (a-r t))}
$$

Donde:

$\mathrm{r}=$ Tasa intrínseca de crecimiento poblacional (día ${ }^{-1}$ )

$\mathrm{N}_{\mathrm{t}}=$ Densidad celular en día $\mathrm{t}\left(\mathrm{cél} \cdot \mathrm{mL}^{-1}\right.$ )

$\mathrm{N}_{\mathrm{o}}=$ Densidad celular en día $1\left(\right.$ cél $\cdot \mathrm{mL}^{-1}$ )

$\mathrm{K}$ = Capacidad de carga (se asumió como la máxima densidad alcanzada) $\mathrm{a}=\ln \left(\left(\mathrm{K}-\mathrm{N}_{\mathrm{o}}\right) / \mathrm{N}_{\mathrm{o}}\right)$

La estimación del parámetro r se realizó mediante minimización de la función de error de los cuadrados residuales, usando la rutina de optimización numérica SOLVER de la hoja de cálculo EXCEL (método del gradiente conjugado, con derivadas centradas).

La tasa de división (div•día ${ }^{-1}$ ) se calculó mediante la fórmula:

Donde:

$$
\mathrm{D}=1 / \mathrm{T}
$$

$\mathrm{T}=$ Tiempo de división (días) = ln (2)/r

\section{Resultados.}

Petróleo crudo

A partir de las concentraciones de 1225, 1750 y $2500 \mathrm{mg} \cdot \mathrm{L}^{-1}$ se observa una inhibición en el crecimiento poblacional dentro de las $24 \mathrm{~h}$ de exposición de $67 \%$, 70 \% y $92 \%$, respectivamente, recuperándose al tercer día de exposición, siendo afectada la tasa de división por día en más del 50 \% con respecto al control (Figura 1).

La tasa intrínseca del crecimiento poblacional (r) para el control fue de 1.56 día $^{-1}$ mientras que para las células expuestas al petróleo crudo estuvo entre 0.75 a 1.19 día $^{-1}$. La tasa de división por día (D) para el control fue de $2.26 \mathrm{div} \cdot$ día $^{-1}$, mientras que para las poblaciones expuestas al petróleo estuvo en rango de 1.08 a $1.71 \mathrm{div} \cdot$ día $^{-1}$ (Tabla 3). Se obtuvo una $\mathrm{CE}_{50 \%}$ de $867.5 \mathrm{mg} \cdot \mathrm{L}^{-1}$ (Figura 2).

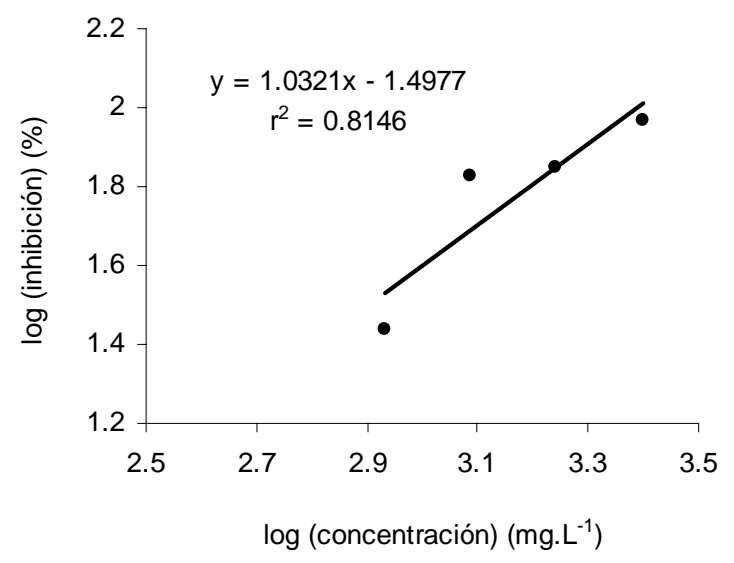

Figura 1. Porcentaje de inhibición del crecimiento poblacional de Chaetoceros gracilis expuesta por 96 horas a una solución con petróleo crudo. 


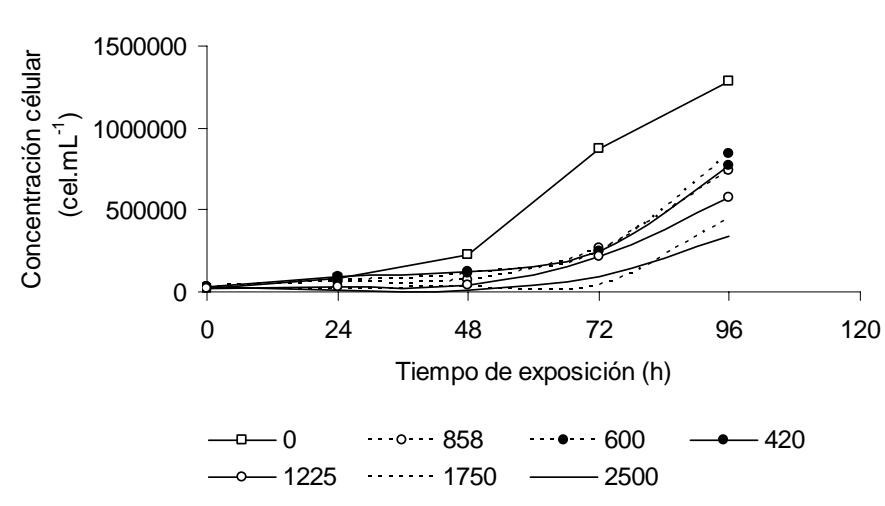

Figura 2. Curvas de crecimiento poblacional de Chaetoceros gracilis expuesta a diferentes concentraciones de petróleo crudo $\left(\mathrm{mg} \cdot \mathrm{L}^{-1}\right)$.

Tabla 3. Tasa intrínseca del crecimiento poblacional (r), tasa de división por día (D) y concentración efectiva media $\left(\mathrm{CE}_{50 \%}\right)$ a $96 \mathrm{~h}$, obtenidas en las pruebas ecotoxicológicas.

\begin{tabular}{cccc}
\hline Agentes tóxicos & $\mathrm{r}\left(\mathrm{dia}^{-1}\right)$ & $\mathrm{D}\left(\mathrm{div} \cdot \mathrm{dia}^{-1}\right)$ & $\begin{array}{c}\mathrm{CE}_{50 \%}\left(\mathrm{mg} \cdot \mathrm{L}^{-1}\right) \\
\mathrm{a} 96 \mathrm{~h}\end{array}$ \\
\hline Petroleo crudo & $0.75-1.19$ & $1.08-1.71$ & 867.5 \\
Kerosene & $0.62-1.48$ & $0.89-2.14$ & 98 \\
Diesel 2 & $0.29-1.44$ & $0.42-2.08$ & 90 \\
\hline
\end{tabular}

\section{Diesel 2}

Concentraciones de Diesel 2 mayores de 196, 280 y $400 \mathrm{mg} \cdot \mathrm{L}^{-1}$ inhibieron el crecimiento de la población expuesta en $97 \%$, $100 \%$ y $100 \%$, respectivamente, dentro de las $24 \mathrm{~h}$ de exposición, no se observó recuperación celular (Figura 3).

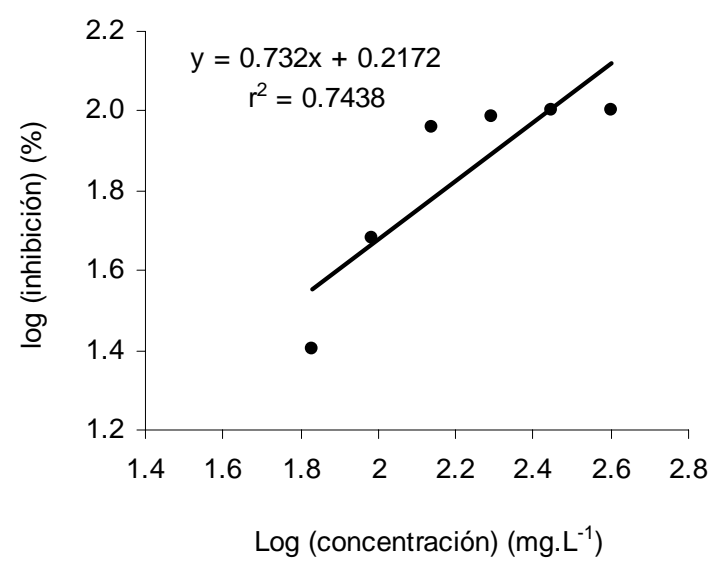

Figura 3. Porcentaje de inhibición del crecimiento poblacional de Chaetoceros gracilis expuesta por 96 horas a una solución con Diesel 2.

La tasa intrínseca del crecimiento poblacional (r) para el control fue de 1.55 día $^{-1}$ mientras que para las poblaciones expuestas a diferentes concentraciones del Diesel 2 estuvo entre 0.62 a 1.48 día $^{-1}$. La tasa de división por día (D) para el control fue de 2.24 div $\cdot d^{-1}{ }^{-1}$, mientras que para las poblaciones expuestas al petróleo estuvo en rango de 0.89 a $2.14 \mathrm{div} \cdot$ día $^{-1}$ (Tabla 3). Se obtuvo una $\mathrm{CE}_{50 \%}$ de $90 \mathrm{mg} \cdot \mathrm{L}^{-1}$ (Figura 4).

Kerosene

Concentraciones de kerosene de $640 \mathrm{y}$ $320 \mathrm{mg} \cdot \mathrm{L}^{-1}$ produjeron una inhibición en el crecimiento poblacional del $96 \%$ y $83 \%$ en el tercer y cuarto día de exposición, respectivamente. Mientras que en concentraciones entre 20 a $160 \mathrm{mg} \cdot \mathrm{L}^{-1}$ causaron una inhibición entre el $8 \%$ al $29 \%$ (Figura 5).

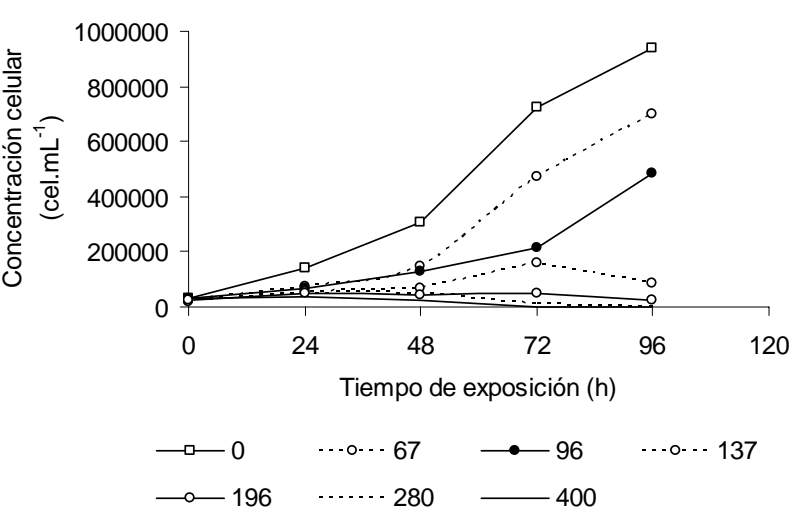

Figura 4. Curvas de crecimiento poblacional de Chaetoceros gracilis expuesta a diferentes concentraciones de Diesel $2\left(\mathrm{mg} \cdot \mathrm{L}^{-1}\right)$.

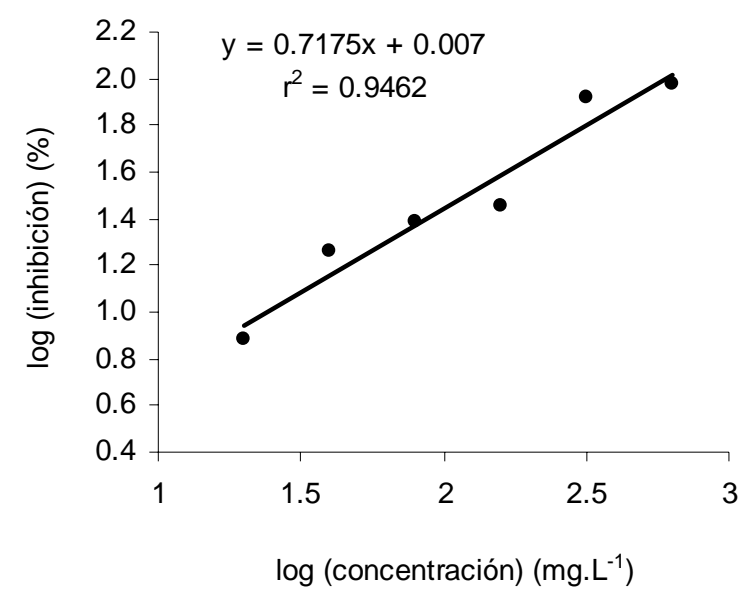

Figura 5. Porcentaje de inhibición del crecimiento poblacional de Chaetoceros gracilis expuesta por 96 horas a una solución con kerosene.

La tasa intrínseca del crecimiento poblacional (r) para el control fue de 1.47 día $^{-1}$ mientras que para las poblaciones expuestas a diferentes concentraciones del kerosene comercial estuvo entre 0.29 a 1.44 día $^{-1}$. La tasa de división por día (D) para el control fue de 2.12 
div $\cdot$ día $^{-1}$, mientras que para las poblaciones expuestas al kerosene estuvo en rango de 0.42 a $2.08 \mathrm{div} \cdot \mathrm{dí}^{-1}$ (Tabla 3). Se obtuvo una $\mathrm{CE}_{50 \%}$ de $98 \mathrm{mg} \cdot \mathrm{L}^{-1}$ (Figura $6)$.

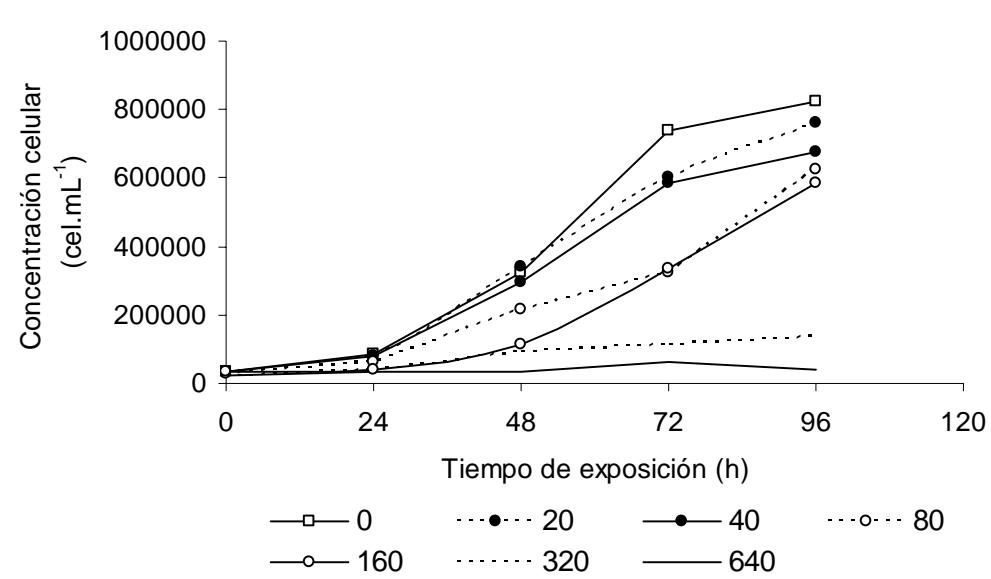

Figura 6. Curvas de crecimiento poblacional de Chaetoceros gracilis expuesta a diferentes concentraciones de kerosene $\left(\mathrm{mg} \cdot \mathrm{L}^{-1}\right)$.

\section{Discusión.}

La compleja composición del petróleo y la extrema variabilidad en la composición del petróleo y los productos refinados del petróleo ha llevado a los investigadores a examinar la toxicidad de una mayor diversidad de ingredientes químicos del petróleo en un esfuerzo por mejorar el entendimiento de las causas de la toxicidad (Wright \& Welbourne, 2002), por tal motivo se consideró importante determinar la toxicidad de los tres productos más usados en el Perú, para consumo doméstico, transporte terrestre $\mathrm{y}$ crudo, el Diesel 2 y el kerosene se encuentran dentro de las primeras 24 horas de exposición, siendo más tóxico el Diesel 2 debido a que presenta mayor concentración de fracciones volátiles, tales como los hidrocarburos alifáticos de bajo peso molecular desde metano hasta octano, que sin embargo son muy volátiles y se pierden rápidamente por evaporación desde la preparación petróleo-agua. Algunos de estos componentes pueden producir efectos narcóticos en animales marinos en concentraciones cerca a su solubilidad acuosa (Crisp et al, 1967). Algo muy semejante ocurre con el kerosene, ésto apoyaría a Craddock (1977 en Neff, 1987) quien señala que la toxicidad aguda se incrementa conforme aumenta la concentración de hidrocarburos aromáticos.

Por otro lado, el petróleo crudo resultó menos tóxico, pues contiene hidrocaburos alifáticos de alto peso molecular que son tan insolubles que no es posible disolverlos suficientemente en agua de mar para causar toxicidad en organismos marinos y éstos finalmente se acumulan en los sedimentos (Crisp et al, 1967).

En las pruebas con petróleo crudo si bien es cierto se produce un efecto inhibitorio a las $24 \mathrm{~h}$, se puede observar una recuperación en la pendiente de crecimiento celular a partir del segundo día,

Tabla 4. Concentración efectiva media $\left(\mathrm{CE}_{50 \%}, 96 \mathrm{~h}\right)$ de hidrocarburos de petróleo y derivados usando diferentes especies acuáticas.

\begin{tabular}{|c|c|c|c|c|}
\hline Organismo prueba & Estadio & Agente tóxico & $\begin{array}{c}\mathrm{CE}_{50 \%} \\
\left(\mathrm{mg} \cdot \mathrm{L}^{-1}\right) \\
96 \mathrm{~h}\end{array}$ & Autor \\
\hline Orthopristis ruber & Juvenil & $\begin{array}{l}\text { Petróleo } \\
\text { liviano }\end{array}$ & 680 & Quevedo et al. (1983) \\
\hline Mugil cephalus & Juvenil & Kerosene & 628.6 & Ibañez \& Huanes (1999) \\
\hline Mugil curema & Juvenil & Petróleo crudo & 1350 & $\begin{array}{l}\text { Aguilera \& Huq (1982 en Ibañez \& Huanes, } \\
\text { 1999) }\end{array}$ \\
\hline Mugil cephalus & Juvenil & Diesel 2 & 694 & Ibañez \& Huanes (1999) \\
\hline Protothaca staminea & Juvenil & Petróleo crudo & 14.7 & Rice et al. (1976 en Weber et al., 2005) \\
\hline Paragrapsus quadridentatus & Adulto & $\begin{array}{l}\text { Petróleo } \\
\text { liviano }\end{array}$ & 2020 & Ahsanullah et al. (1982 en Weber et al., 2005) \\
\hline Paralithodes camtschatica & Larva & Petróleo crudo & 5 & Rice et al. (1976 en Weber et al., 2005) \\
\hline Acanthomysis pseudomacropsis & Larva & Petróleo crudo & 8.99 & Rice et al. (1976 en Weber et al., 2005) \\
\hline Orchomene pinguis & Juvenil & Petróleo crudo & 7.4 & Rice et al. (1976 en Weber et al., 2005) \\
\hline Chaetoceros gracilis & Adulto & Petróleo crudo & 867.5 & Vera et al. (presente estudio) \\
\hline Chaetoceros gracilis & Adulto & Diesel 2 & 90 & Vera et al. (presente estudio) \\
\hline Chaetoceros gracilis & Adulto & Kerosene & 98 & Vera et al. (presente estudio) \\
\hline
\end{tabular}


et al. (1988) quien determinó en otras condiciones de temperatura y salinidad un crecimiento celular después del segundo día tendiendo a igualar el crecimiento del cultivo control y después del tercer día parece estar estimulado, superando al cultivo control. Después de la volatilización de los hidrocarburos aromáticos hay una tendencia a la recuperación en los 3 casos, especialmente en los cultivos que tienen las más bajas concentraciones. Esto también explicaría la mayor toxicidad del petróleo crudo en comparación con Diesel 2 y Diesel 6 encontrada por Alayo \& Iannacone (2002) usando rotíferos en pruebas de 48 h.

Comparando los resultados obtenidos en el presente estudio para la especie $C h$. gracilis, con los resultados en otras especies (Tabla 4), se obtuvo las siguientes secuencias de sensibilidad:

- Petróleo crudo: Amphipoda (Orchomene pinguis) > Microalga (Chaetoceros gracilis) > Pez (Mugil curema) > Decapoda (Paragrapsus quadridentatus)

- Diesel: Microalga (Chaetoceros gracilis) > Pez (Mugil curema)

- Kerosene: Microalga (Chaetoceros gracilis) > Pez (Mugil curema)

En el Perú, la Ley General de Aguas vigente (MINSA, 1969) no considera estándares de calidad para los hidrocarburos de petróleo. Sin embargo, en Alaska, el estándar de calidad acuática marina para el crecimiento y propagación de peces, mariscos, vida acuática y vida silvestre es de $15 \mu \mathrm{g} \cdot \mathrm{L}^{-1}$ para hidrocarburos acuosos totales (HAT) (ADEC, 2003).

\section{Conclusiones.}

Las soluciones con petróleo crudo, Diesel 2 y kerosene produjeron un efecto de inhibición sobre el crecimiento poblacional de la diatomea $C h$. gracilis, siendo las concentraciones efectivas media (CE50\%) de 867.5, 90 y $98 \mathrm{mg} \cdot \mathrm{L}^{-1}$, respectivamente. Este efecto inhibidor fue mayor dentro de las $24 \mathrm{~h}$, siendo afectadas la tasa intrínseca de crecimiento y la tasa de división por día. La recuperación celular fue notable a partir del tercer día con una tendencia a alcanzar los cultivos controles en los días posteriores.

Ch. gracilis fue una especie sensible a los derivados aromáticos del hidrocarburo de petróleo (petróleo crudo, Diesel 2 y kerosene), por lo que puede ser utilizada como organismo prueba para evaluar la toxicidad de otros compuestos orgánicos acuáticos marinos, siguiendo las condiciones recomendadas.

Se recomienda realizar estudios subletales para observar los efectos en largos periodos de tiempo, para evaluar el daño potencial causado por un evento de derrame o una descarga crónica y para predecir el valor de recuperación del medio afectado o impactado.

\section{Agradecimientos.}

Se agradece al Sr. José Ortega por el apoyo brindado durante la parte experimental del trabajo. Asimismo, agradecemos al M.Sc. Ricardo Oliveros por la revisión del manuscrito.

\section{Literatura citada.}

Alaska Department of Environmental Conservation (ADEC). 2003. Water quality standards. 18 AAC 70.

Alayo M. \& Iannacone J. 2002. Ensayos ecotoxicológicos con petroleo crudo, Diesel 2 y Diesel 6 con dos subespecies de Brachionus plicatilis Müller 1786 (Rotifera: Monogononta). Gayana. 66: 45-58.

Alayo M., Iannacone J. \& Arrascue A. 2004. Sensibilidad al cromo: microbiopruebas con las diatomeas marinas Isochrysis galbana Parke y Chaetoceros gracilis Schütt. Ecol. Apl. 3: 154-161.

Cabello R., Jacinto M.E., Morón O. y Chang F. 1999. Evaluación de la calidad medio marino costanero en la Bahía de Talara y Aguas Adyacentes, 15-17 Abril 1997. Inf. Prog. Inst. Mar Perú. 106.

Crisp D.J., Christhie O. \& Ghobashy A. 1967. Narcotic and toxic action of organic compounds on barnacle larvae. Comp. Biochem. Physiol. 22: 629-649.

D' Croz L., Torres J. \& Gómez J. 1988. Efecto del crudo venezolano (BCF-24) sobre el crecimiento de la diatomea marina tropical Chaetoceros gracilis (Thomas, 1966). Rev. Pacífico Sur. (Número Especial).: 171-178.

Franco J. 1985. Manual de ecología. Ed. Trillas, México.

González M., Parra O. \& Cifuentes A. 1995. Técnicas de cultivo de microalgas en laboratorio. : 219-250. En: Alveal, K. y M. E. Ferrario. (Eds.). Manual de métodos ficológicos. Universidad de Concepción.

Guzmán, M. 1995. Evaluación de la contaminación marina frente a la Bahía de Paita. Crucero Demersales 950506. Inf. Prog. Inst. Mar Perú. (7):1-16.

Guzmán M., Chávez J., Morón O., Sánchez S., \& Flores G. 1997. Evaluación de la calidad del medio ambiente marino en la bahía de Pisco - Paracas, 22 a 24 mayo 1996. Inf. Prog. Inst. Mar Perú. 54: 3-29.

Ibañez J. \& Huanes L. 1999. Bioensayos de toxicidad letal de gasolina $84^{\circ}$, kerosene industrial, petróleo diesel 2 y petróleo diesel 6 en lisa Mugil cephalus L. II Congreso Peruano de Ecología, Medio Ambiente y Desarrollo, Huancayo, Perú.

Jacinto M.E. \& Cabello R. 1996. Niveles de hidrocarburos de petróleo en el ecosistema marino costero del Perú. Bahías seleccionadas. Periodo 1996. Inf. Prog. Inst. Mar. Perú. (110):1-60.

Jacinto M.E., Cabello R., Guzmán M., Morón O., Villanueva P. \& Córdova J. 1996. Evaluación de la contaminación marina en la Bahía Ferrol, Chimbote. 14-18 Julio 1994. Inf. Prog. Inst. Mar Perú. (48):21-56.

Jacinto M.E., Morón O., Martínez C., Villanueva P., Guzmán M. \& Córdova J. 1997. Evaluación de la calidad del medio ambiente marino en el área de Bayóvar-Ensenada de Sechura, 8 al 10 mayo 1996. Inf. Prog. Inst. Mar Perú. (54):30-64.

Jacinto, M.E., Chávez J., Morón O., Sánchez S. \& Solís J. 1998. Evaluación de la calidad del medio marino en Bahía Supe-Paramonga en enero 1997. Inf. Prog. Inst. Mar Perú. (80):15-35. 
Joubert G. 1980. A bioassay application for quantitative toxicity measurements using the green algae Selenastrum capricornutum. Water Res. 14: 17591763.

Ministerio de Energía y Minas (MEM). 2006. Lotes con contratos para operaciones petroleras en el Perú. Atlas minería y energía en el Perú 2001. En línea: http://intranet2.minem.gob.pe/web/archivos/ogp/public aciones/atlasmineriaenergia2001/hidrocarburos/2001_l otes_cont_oper_petro.pdf.

Ministerio de Salud (MINSA). 1969. Ley General de Aguas (D.L. 17752). El Peruano.

Neff J. 1987. Biological Effects of Oil in the Marine Environment. Chem. Engineering Progress. Nov. : $27-$ 33

Ochoa N., Gómez O., Sánchez S. \& Delgado E. 1999. Diversidad de diatomeas y dinoflagelados marinos del Perú. Bol. Inst. Mar Perú. 18: 1-14.

Quevedo J., Segovia J., Pérez J. \& Zurburg W. 1983. Efectos del petróleo y algunos dispersantes en juveniles del Cococoro, Orthopristis ruber (Pisces: Pomadasyidae). Bol. Inst. Oceanogr. de Venez. Univ. de Oriente. 22: 177-184.
Sánchez G. \& Orozco R. 1997. Diagnóstico Regional sobre actividades realizadas en tierra que afectan los ambientes marino, costero y dulceacuicolas asociados al Pacífico Sudeste. Informe de Consultoría para la Comisión Permanente del Pacífico Sur (CPPS) del Programa de las Naciones Unidad para el Medio Ambiente.

Vera G., Tam J., Pinto E. \& Angulo J. 2001. Efecto del cadmio sobre el crecimiento poblacional de la diatomea marina Chaetoceros gracilis Schutt. Rev. Peru. Biol. 8:45-52.

Vizcarra A. 2002. Ecósfera. La Ciencia ambiental y los desastres ecológicos. Editorial. Siglo XXI.

Weber C.I. 1993. Methods for measuring the acute toxicity of effluents and receiving waters to freshwater and marine organisms. EPA600490027F. 293 p.

Weber P., Dasher D., Duffy L., Perkins R. \& O'Hara T. 2005. Acute and chronic toxicity of hydrocarbons in marine and fresh water with an emphasis on Alaska species: a review of the literature. Alaska Dept. Env. conservation.

Wright D.A. \& Welbourne P. 2002. Environmental toxicology. Cambridge Univ. Press.

1 Afiliación en el momento de la investigación: Instituto del Mar del Perú, Esq. Gamarra y Gral. Valle s/n, La Punta, Callao. Afiliación actual: Universidad Ricardo Palma, Benavides 5440, Santiago de Surco, gioverad@yahoo.com.

2 Instituto del Mar del Perú, Esq. Gamarra y Gral. Valle s/n, La Punta, Callao, jtam@imarpe.gob.pe.

3 Instituto del Mar del Perú, Esq. Gamarra y Gral. Valle s/n, La Punta, Callao, epinto@imarpe.gob.pe. 